\title{
Diabetic Ketoacidosis-Associated Stroke in Children and Youth
}

\author{
Jennifer Ruth Foster, ${ }^{1,2}$ Gavin Morrison, ${ }^{1}$ and Douglas D. Fraser ${ }^{1,2,3,4,5,6}$ \\ ${ }^{1}$ Critical Care Medicine and Paediatrics, University of Western Ontario, London, ON, Canada N6A 5W9 \\ ${ }^{2}$ Children's Health Research Institute, London, ON, Canada N6C 2 V5 \\ ${ }^{3}$ Centre for Critical Illness Research, London, ON, Canada N6C 2 V5 \\ ${ }^{4}$ Clinical Neurological Sciences, University of Western Ontario, London, ON, Canada N6A 3K7 \\ ${ }^{5}$ Physiology and Pharmacology, University of Western Ontario, London, ON, Canada N6A 3K7 \\ ${ }^{6}$ Translational Research Centre, London, ON, Canada N6C 2V5
}

Correspondence should be addressed to Jennifer Ruth Foster, jennifer.foster@lhsc.on.ca

Received 8 October 2010; Revised 12 November 2010; Accepted 4 December 2010

Academic Editor: Halvor Naess

Copyright (C) 2011 Jennifer Ruth Foster et al. This is an open access article distributed under the Creative Commons Attribution License, which permits unrestricted use, distribution, and reproduction in any medium, provided the original work is properly cited.

\begin{abstract}
Diabetic ketoacidosis (DKA) is a state of severe insulin deficiency, either absolute or relative, resulting in hyperglycemia and ketonemia. Although possibly underappreciated, up to $10 \%$ of cases of intracerebral complications associated with an episode of DKA, and/or its treatment, in children and youth are due to hemorrhage or ischemic brain infarction. Systemic inflammation is present in DKA, with resultant vascular endothelial perturbation that may result in coagulopathy and increased hemorrhagic risk. Thrombotic risk during DKA is elevated by abnormalities in coagulation factors, platelet activation, blood volume and flow, and vascular reactivity. DKA-associated cerebral edema may also predispose to ischemic injury and hemorrhage, though cases of stroke without concomitant cerebral edema have been identified. We review the current literature regarding the pathogenesis of stroke during an episode of DKA in children and youth.
\end{abstract}

\section{Introduction}

Type 1 diabetes mellitus (T1DM) is a common autoimmune condition that often presents in childhood and may be complicated by episodes of diabetic ketoacidosis (DKA). DKA is a state of severe insulin deficiency, either absolute or relative, resulting in hyperglycemia, ketonemia, acidemia, and systemic inflammation. Compared with adults, episodes of DKA in children carry a higher risk of morbidity and mortality. This is predominantly attributable to intracerebral complications [1-5], which occur in 3-10 pediatric patients per 1000 cases of DKA [6]. The most common intracerebral complication of DKA is cerebral edema (DKA-CE), which results in the death of $21-24 \%$ of affected patients, and significant morbidity in a further 10-35\% [6-8]. Less common, and perhaps underappreciated, is the risk of acute ischemic or hemorrhagic stroke during the acute DKA episode. It has been estimated based on case series that approximately $10 \%$ of intracerebral complications of DKA are due to hemorrhage or ischemic brain infarction $[4,5]$. While some cases of brain infarction may arise secondary to DKA-CE-induced herniation with resultant vessel occlusion, it has become clear that not all cases of stroke in DKA are associated with cerebral edema (CE). As the presentation of stroke associated with DKA may mimic that of CE but requires different management strategies, it is imperative for the clinician to be cognizant of this potential complication. This review will examine the etiology and pathogenesis of stroke associated with episodes of DKA in children and youth (age <20). While Type 2 diabetes mellitus is becoming more commonly recognized in the pediatric population and may present with, or be complicated by, DKA $[9,10]$, cerebral thrombosisassociated stroke has not been reported with Type 2 diabetes mellitus. Thus, the review focuses on children with T1DM.

\section{Pathogenesis of Ischemic Stroke}

Diabetes mellitus is a known independent risk factor for ischemic stroke, conferring two times the risk of an ischemic event in adults compared to the nondiabetic population 
$[11,12]$. There are several characteristics of DKA that place children at higher risk of cerebral ischemia. The reported cases on DKA-associated stroke in children and youth are presented in Table 1.

2.1. DKA as a Systemic Inflammatory Illness. DKA is more than simply a deterioration of glucose metabolism; it is also associated with a systemic inflammatory response characterized by vascular endothelial injury and coagulopathy. The inflammatory state accompanying DKA is characterized by elevated levels of inflammatory markers (CRP), cytokines (IL6, IL1 $\beta, \mathrm{TNF} \alpha$ ), and complement activation [26-30]. It is likely that the oxidative stress induced by hyperglycemia and ketosis [31] contributes to this inflammatory reaction and results in diffuse vascular injury. Evidence of vascular endothelial injury can be seen in pretreatment subclinical CE [1], pulmonary interstitial edema $[2,23,24,32]$, disseminated intravascular coagulation (DIC) $[13,16,33$, 34], and elevated levels of thrombomodulin [35].

Chronically, the vascular endothelium is a primary target of the abnormal glycemic metabolism in T1DM [27]. Children with T1DM may be at risk of a chronic state of inflammation and endothelial activation outside of episode of DKA. Children within 1 year of diagnosis have been reported to have biochemical evidence of inflammation, with increased levels of both serum prothrombin fragments and TNF $\alpha$ compared to children more than 1 year post-diagnosis and to nondiabetic controls [36]. Furthermore, this report detailed evidence of endothelial perturbation, characterized by levels of von Willebrand Factor (vWF) and tissue plasminogen activator (tPA) more than 2 standard deviations higher than control. Another study found that the endothelial cellspecific adhesion molecule, soluble endothelial leukocyte adhesion molecule (sE-Selectin), was elevated in children with T1DM compared to healthy controls, and positively correlated with serum glucose concentration [37]. Analysis of the coagulation system in adults with diabetes has also identified abnormalities in many steps of the coagulation system [38].

2.2. Abnormalities in the Coagulation Cascade. Two case controlled studies $[42,43]$ found an increased rate $(50 \%)$ of clinically apparent deep venous thrombosis (DVT) in very young children (less than 3 years) with DKA who required femoral central venous catheter (CVC) insertion when compared with age-matched nondiabetic controls (who also underwent femoral CVC insertion). Comparatively, an incidence rate of $1.5-18.3 \%$ has been described for clinically or radiologically apparent femoral CVC-associated DVTs in the PICU population $[42,44]$. Although the propensity for hypercoagulability in diabetes mellitus has not been described as a specific isolated risk factor for DVT in children [42], it is clear that the procoagulant mechanisms that place children with an episode of DKA at risk of CVC-related DVT may also act to increase the risk of stroke.

The inflammatory condition seen in DKA, with endothelial perturbation, predisposes to an acquired procoagulant state [26]. While the majority of case studies of children with DKA-associated stroke have not identified consistent, generalized alterations in the coagulation system (Table 1), these studies have mainly examined the coagulation system at a single time point after identification of a neurologic abnormality. More systematic evaluation of coagulation abnormalities during an episode of DKA requires longitudinal consideration, both before and during therapeutic intervention. Indeed, longitudinal studies in children [41] and adults [35] with DKA have identified multiple coagulation abnormalities, including increased platelet aggregation, elevated levels of procoagulants, and decreased activity of anticoagulants. Coagulation factors for which abnormalities have been noted during DKA or its treatment in either children or adults have been summarized (Table 2).

Examination of the coagulation factors of 7 adolescents on presentation with DKA and at several time points after initiation of DKA therapy demonstrated abnormalities in Protein C, Protein S, plasma homocysteine, and von Willebrand Factor (vWF) [41]. Protein C levels are initially elevated but quickly decrease to normal with DKA treatment, while Protein $\mathrm{C}$ activity is initially low and slowly normalizes with treatment. Adult patients with T1DM have significantly lower protein C levels than controls [45]. Their protein C levels are inversely related to glucose concentrations but exhibit no relationship with glycosylated hemoglobin A (HbAlc) levels. This latter finding suggests that acute, rather than chronic, variations in blood glucose may determine the response of Protein $\mathrm{C}$, which may itself explain the normal values found in most patients with DKA-associated stroke (Table 1).

Plasma homocysteine is an important factor in atherosclerosis and thrombosis [46] and also decreases protein C activation [47]. Plasma homocysteine levels in adolescents are elevated in DKA and gradually normalize after insulin initiation [41]. The relatively rapid homocysteine rise may result in the very gradual normalization of protein $\mathrm{C}$ activity that has been observed. In adolescents, Protein $S$ antigen levels remain normal during DKA while free protein $\mathrm{S}$, the active anticoagulant, is reduced and does not return to baseline with treatment [41]. This is consistent with the finding that low levels of free protein $S$ are the result of increased levels of C4b-binding protein in poorly controlled adult T1DM patients [30].

vWF is synthesized and secreted by endothelial cells, facilitates platelet adhesion, and is a carrier protein for factor VIII. High vWF levels are a marker of endothelial injury and activation. In adolescents, vWF antigen and activity are initially increased in DKA and decrease slowly with DKA therapy [41]. Factor VIII concentration is also elevated in adults with long-standing insulin-dependent diabetes mellitus during an episode of DKA [48, 49]. The fibrinogen concentration remains normal throughout DKA and its management [41]. However, fibrinogen circulating in an environment of high glucose can become hyperglycosylated [50] with resulting fibrin fibers that are resistant to plasmin degradation [51].

In 8 adults receiving a continuous subcutaneous insulin infusion, researchers examined the effects of infusion cessation for 4 hours [40]. All subjects entered early biochemical DKA. Tests of fibrinolytic activity after vascular stimulation 
TABLE 1: Case reports of arterial ischemic stroke, cerebral venous thrombotic stroke, and hemorrhagic stroke associated with an episode of DKA in children and youth.

\begin{tabular}{|c|c|c|c|c|c|c|c|}
\hline $\begin{array}{l}\text { Patient } \\
\text { number }\end{array}$ & $\begin{array}{l}\begin{array}{l}\text { Age } \\
\text { (year) }\end{array} \\
\end{array}$ & Gender & Pathologic findings & Clinical Presentation & Outcome & Coagulation profile & $\begin{array}{c}\text { Reference } \\
\text { number }\end{array}$ \\
\hline \multicolumn{8}{|c|}{ Arterial Ischemic Stroke. } \\
\hline 1 & 0.25 & female & $\begin{array}{l}\text { Multiple small vessel } \\
\text { thrombi with edema } \\
\text { on autopsy }\end{array}$ & $\begin{array}{l}\text { First presentation } \\
\text { diabetes, generalized } \\
\text { seizure, progressive } \\
\text { coma on admission } \\
\end{array}$ & Death at 24 hours & & {$[13]$} \\
\hline 2 & 4 & female & $\begin{array}{l}\text { Infarction, right } \\
\text { posterior cerebral } \\
\text { artery distribution }\end{array}$ & $\begin{array}{l}\text { First presentation } \\
\text { diabetes, decerebrate } \\
\text { posturing, acute } \\
\text { herniation }\end{array}$ & $\begin{array}{l}\text { Slowly regained ability } \\
\text { to walk and } \\
\text { comprehend speech }\end{array}$ & $\begin{array}{l}\text { Low protein C } \\
\text { normalized with } \\
\text { treatment, elevated } \\
\text { Factor VIII-vWF } \\
\text { complex, elevated } \\
\text { plasma and platelet } \\
\text { thromboxane B2 }\end{array}$ & {$[14]$} \\
\hline 3 & 8 & male & $\begin{array}{l}\text { Infarction of left } \\
\text { thalamus, left } \\
\text { temporal lobe, B/L } \\
\text { occipital lobes }\end{array}$ & Decerebrate posturing & Slow recovery & $\begin{array}{l}\text { Low protein } C \\
\text { antigen, } \\
\text { normalized }\end{array}$ & {$[14]$} \\
\hline 4 & 10 & unknown & $\begin{array}{l}\text { Basilar artery } \\
\text { thrombosis on CT }\end{array}$ & $\begin{array}{l}\text { Restless, decreasing } \\
\text { LOC over } 4.5 \text { hours, } \\
\text { respiratory arrest at } 7 \\
\text { hours }\end{array}$ & $\begin{array}{l}\text { Persistent vegetative } \\
\text { state }\end{array}$ & & {$[4]$} \\
\hline 5 & 14 & female & $\begin{array}{l}\text { CT edema and } \\
\text { infarction of left } \\
\text { lentiform nucleus, } \\
\text { thalami, B/L } \\
\text { peduncles }\end{array}$ & $\begin{array}{l}\text { Headache, } \\
\text { deteriorating LOC. } \\
\text { Pupils midline, fixed, } \\
\text { dilated after } 12 \text { hours }\end{array}$ & $\begin{array}{l}\text { Mild left hemiparesis, } \\
\text { behavioral } \\
\text { disturbances }\end{array}$ & & {$[15]$} \\
\hline 6 & 5 & male & $\begin{array}{l}\text { Infarction left } \\
\text { posterior cerebral } \\
\text { artery distribution, } \\
\text { geniculate nuclei, left } \\
\text { thalamus }\end{array}$ & $\begin{array}{l}\text { First presentation } \\
\text { diabetes, generalized } \\
\text { seizure }\end{array}$ & $\begin{array}{l}\text { Moderate left } \\
\text { hemiplegia }\end{array}$ & $\begin{array}{l}\text { Low protein } \mathrm{S} \text {, } \\
\text { elevated factor VIII } \\
\text { and factor } \mathrm{V}\end{array}$ & {$[5]$} \\
\hline 7 & 6 & male & $\begin{array}{l}\text { Infarction } \mathrm{B} / \mathrm{L} \\
\text { anterior cerebral } \\
\text { artery distributions, } \\
\text { basal ganglia, left } \\
\text { cingulate gyrus } \\
\end{array}$ & $\begin{array}{l}\text { First presentation } \\
\text { diabetes, lethargy and } \\
\text { posturing of upper } \\
\text { extremities }\end{array}$ & $\begin{array}{l}\text { Emotionally labile, } \\
\text { intellectual and motor } \\
\text { impairment }\end{array}$ & $\begin{array}{l}\text { Low AT III antigen, } \\
\text { AT III functionally } \\
\text { normal, increased } \\
\text { platelet } \\
\text { aggregation }\end{array}$ & {$[5]$} \\
\hline 8 & 7 & male & $\begin{array}{l}\text { Ischemia in globus } \\
\text { pallidus, } \mathrm{B} / \mathrm{L} \\
\text { cingulate gyri. } \\
\text { Infarctions left } \\
\text { thalamus, right } \\
\text { medial occipital lobe. } \\
\text { No CT edema }\end{array}$ & $\begin{array}{l}\text { First presentation } \\
\text { diabetes, decreased } \\
\text { level of consciousness } \\
\text { with incontinence, } \\
\text { stiffness, pupils poorly } \\
\text { reactive }\end{array}$ & $\begin{array}{l}\text { Hemiplegia, normal } \\
\text { cognition, abnormal } \\
\text { behavior and affect, }\end{array}$ & $\begin{array}{l}\text { Decreased platelet } \\
\text { aggregation }\end{array}$ & {$[5]$} \\
\hline 9 & 8 & male & $\begin{array}{l}\text { Infarction thalamus, } \\
\text { midbrain, basal } \\
\text { ganglia, cingulated } \\
\text { gyrus. No CT edema }\end{array}$ & $\begin{array}{l}\text { First presentation } \\
\text { diabetes, unresponsive, } \\
\text { flaccid, pupils dilated }\end{array}$ & Vegetative state & $\begin{array}{l}\text { Low aPTT ( } 21 \\
\text { seconds) }\end{array}$ & {$[5]$} \\
\hline 10 & 10 & male & $\begin{array}{l}\text { Infarction right } \\
\text { anterior cerebral } \\
\text { artery distribution, } \\
\text { left putamen, B/L } \\
\text { globus pallidus }\end{array}$ & $\begin{array}{l}\text { First presentation } \\
\text { diabetes, decreased } \\
\text { LOC, left extensor } \\
\text { posturing, abnormal } \\
\text { papillary response }\end{array}$ & $\begin{array}{l}\text { Severe focal neurologic } \\
\text { impairment }\end{array}$ & & {$[5]$} \\
\hline 11 & 6 & female & $\begin{array}{l}\text { Infarction proximal } \\
\text { left middle cerebral } \\
\text { artery, left basal } \\
\text { ganglia }\end{array}$ & $\begin{array}{l}\text { First presentation } \\
\text { diabetes, irritability, } \\
\text { lethargy, right } \\
\text { hemiparesis, aphasia. } \\
\text { Had } 2 \text { mitral valve } \\
\text { thrombi }\end{array}$ & $\begin{array}{l}\text { Regained speech, } \\
\text { residual right } \\
\text { hemiparesis }\end{array}$ & $\begin{array}{l}\text { Normal } \\
\text { pro-thrombotic } \\
\text { studies }\end{array}$ & {$[6]$} \\
\hline
\end{tabular}


TABle 1: Continued.

\begin{tabular}{|c|c|c|c|c|c|c|c|}
\hline $\begin{array}{l}\text { Patient } \\
\text { number }\end{array}$ & $\begin{array}{l}\text { Age } \\
\text { (year) }\end{array}$ & Gender & Pathologic findings & Clinical Presentation & Outcome & Coagulation profile & $\begin{array}{c}\text { Reference } \\
\text { number }\end{array}$ \\
\hline 12 & 18 & female & $\begin{array}{l}\text { Infarction right } \\
\text { common carotid } \\
\text { artery territory with } \\
\text { distal emboli in right } \\
\text { anterior and middle } \\
\text { cerebral arteries }\end{array}$ & $\begin{array}{l}\text { First presentation } \\
\text { diabetes, left } \\
\text { hemiparesis } 10 \text { hours } \\
\text { after carotid artery } \\
\text { puncture }\end{array}$ & $\begin{array}{l}\text { Moderate clinical } \\
\text { recovery }\end{array}$ & & {$[16]$} \\
\hline \multicolumn{8}{|c|}{ Cerebral Venous Thrombosis. } \\
\hline 13 & 5 & female & $\begin{array}{l}\text { Thrombosis of } \\
\text { straight sinus and } \\
\text { vein of Galen. } \\
\text { Infarction of basal } \\
\text { ganglia, thalamus }\end{array}$ & $\begin{array}{l}\text { Confusion, decreased } \\
\text { LOC, rigidity, fisting. } \\
\text { Significant iron } \\
\text { deficiency anemia }\end{array}$ & $\begin{array}{l}\text { Mild learning } \\
\text { difficulties }\end{array}$ & $\begin{array}{l}\text { Normal clotting } \\
\text { screen and } \\
\text { thrombophilia } \\
\text { screen }\end{array}$ & {$[17]$} \\
\hline 14 & 11 & male & $\begin{array}{l}\text { Multiple areas of } \\
\text { infarction on MRI } \\
\text { without hemorrhage } \\
\text { or edema }\end{array}$ & $\begin{array}{l}\text { Headache, nausea and } \\
\text { vomiting, acute } \\
\text { deterioration with } \\
\text { fixed, dilated pupils. } \\
\text { Had DVT of right } \\
\text { superficial femoral and } \\
\text { popliteal veins }\end{array}$ & Brain death & $\begin{array}{l}\text { Decreased protein } \\
\text { C function }(36 \%) \text {, } \\
\text { normal protein S } \\
\text { and factor VIII, no } \\
\text { anticardiolipins. } \\
\text { Heterozygous } \\
\text { factor V Leiden }\end{array}$ & {$[18]$} \\
\hline 15 & 19 & female & $\begin{array}{l}\text { Superior sagittal sinus } \\
\text { thrombosis }\end{array}$ & $\begin{array}{l}\text { First presentation } \\
\text { diabetes. Anxiety } \\
\text { progressed to } \\
\text { psychosis, dysphasia, } \\
\text { left abducens palsy, } \\
\text { right inferior facial } \\
\text { palsy, tetraparCsis with } \\
\text { upper motor neuron } \\
\text { signs }\end{array}$ & $\begin{array}{l}\text { Partial left abducens } \\
\text { paresis with diplopia } \\
\text { which resolved }\end{array}$ & $\begin{array}{l}\text { coagulopathy } \\
\text { screen negative }\end{array}$ & [19] \\
\hline 16 & 8 & male & $\begin{array}{l}\text { Vein of galen and } \\
\text { superior sagittal sinus } \\
\text { thrombosis. B/L } \\
\text { medial cerebral } \\
\text { hemisphere } \\
\text { infarctions }\end{array}$ & $\begin{array}{l}\text { First presentation } \\
\text { diabetes, loss of } \\
\text { consciousness, sluggish } \\
\text { pupillary reaction, } \\
\text { fever }\end{array}$ & $\begin{array}{l}\text { GCS remained } 6 \text { when } \\
\text { transferred to another } \\
\text { hospital }\end{array}$ & $\begin{array}{l}\text { Low platelets, } \\
\text { decreased ATIII } \\
(60.4 \%) \text { increased } \\
\text { with treatment, } \\
\text { elevated D-dimer, } \\
\text { increased with } \\
\text { treatment }\end{array}$ & {$[20]$} \\
\hline 17 & 1.1 & female & $\begin{array}{l}\text { Left transverse sinus } \\
\text { thrombosis, no } \\
\text { infarction }\end{array}$ & $\begin{array}{l}\text { First presentation of } \\
\text { thiamine-responsive } \\
\text { megaloblastic anemia, } \\
\text { associated with } \\
\text { nonimmune } \\
\text { insulin-dependent } \\
\text { diabetes. Right-sided } \\
\text { focal seizure }\end{array}$ & $\begin{array}{l}\text { Normal neurologic } \\
\text { status }\end{array}$ & $\begin{array}{l}\text { Prothrombotic } \\
\text { screening negative }\end{array}$ & {$[16]$} \\
\hline 18 & 10 & female & $\begin{array}{l}\text { Thrombosis of } \\
\text { superior sagittal, } \\
\text { straight, right } \\
\text { transverse, right } \\
\text { sigmoid and proximal } \\
\text { posterior left } \\
\text { transverse sinuses }\end{array}$ & $\begin{array}{l}\text { Headache, 6th cranial } \\
\text { nerve palsy day } 3, \\
\text { decreased level of } \\
\text { consciousness day } 5\end{array}$ & $\begin{array}{l}\text { Recombinant tPA } \\
\text { thrombolysis, complete } \\
\text { recovery }\end{array}$ & $\begin{array}{l}\text { Heterozygous } \\
\text { mutation of the } \\
\text { prothrombin gene } \\
\text { (G20210A) }\end{array}$ & {$[21]$} \\
\hline \multicolumn{8}{|c|}{ Hemorrhagic infarction. } \\
\hline 19 & 11 & female & $\begin{array}{l}\text { Multiple large, } \mathrm{B} / \mathrm{L} \\
\text { posterior temporal } \\
\text { lobe hematomas }\end{array}$ & $\begin{array}{l}\text { Behavioral disturbance, } \\
\text { lethargy, progressed to } \\
\text { unresponsive, pupils } \\
\text { dilated and unreactive }\end{array}$ & $\begin{array}{l}\text { Normal neurologic } \\
\text { exam }\end{array}$ & & {$[22]$} \\
\hline 20 & 1 & unknown & $\begin{array}{l}\text { Subarachnoid } \\
\text { hemorrhage on CT }\end{array}$ & $\begin{array}{l}\text { Sudden respiratory } \\
\text { arrest }\end{array}$ & Died at 2 days & & {$[4]$} \\
\hline
\end{tabular}


TABle 1: Continued.

\begin{tabular}{|c|c|c|c|c|c|c|c|}
\hline $\begin{array}{l}\text { Patient } \\
\text { number }\end{array}$ & $\begin{array}{l}\begin{array}{l}\text { Age } \\
\text { (year) }\end{array} \\
\end{array}$ & Gender & Pathologic findings & Clinical Presentation & Outcome & Coagulation profile & $\begin{array}{c}\text { Reference } \\
\text { number }\end{array}$ \\
\hline 21 & 11 & unknown & $\begin{array}{l}\text { Subarachnoid } \\
\text { hemorrhage on CT }\end{array}$ & $\begin{array}{l}\text { Progressively } \\
\text { worsening neurologic } \\
\text { status }\end{array}$ & Death & & [4] \\
\hline 22 & 6.5 & unknown & $\begin{array}{l}\text { CT suggestive of } \\
\text { subarachnoid } \\
\text { hemorrhage }\end{array}$ & $\begin{array}{l}\text { Severe headache and } \\
\text { restless. Pupils fixed, } \\
\text { dilated at } 3 \text { hours, } \\
\text { respiratory arrest at } 6 \\
\text { hours }\end{array}$ & Death & & {$[4]$} \\
\hline 23 & 9 & female & $\begin{array}{l}\text { Hemorrhagic } \\
\text { infarction right } \\
\text { caudate nucleus, } \\
\text { anterior limb of } \\
\text { internal capsule. } \\
\text { Non-hemorrhagic } \\
\text { infarction of } B / \mathrm{L} \\
\text { thalami with edema }\end{array}$ & $\begin{array}{l}\text { Ataxia, deteriorating } \\
\text { LOC, abnormal } \\
\text { respiratory pattern,. } \\
\text { Developed decorticate } \\
\text { posturing, right-sided } \\
\text { tonic seizure }\end{array}$ & $\begin{array}{l}\text { Communication } \\
\text { disorder, asymmetric } \\
\text { spastic quadriparesis, } \\
\text { behavior disturbances. }\end{array}$ & & {$[15]$} \\
\hline 24 & 9 & female & $\begin{array}{l}\text { Edema and } \\
\text { hemorrhagic } \\
\text { infarctions basal } \\
\text { ganglia, upper brain } \\
\text { stem, medial } \\
\text { temporal lobes, } \\
\text { frontal lobes, occipital } \\
\text { lobes }\end{array}$ & $\begin{array}{l}\text { Decreased LOC, left } \\
\text { exotropia, unequal and } \\
\text { unreactive pupils, } \\
\text { papilledema }\end{array}$ & $\begin{array}{l}\text { Quadriplegia, absent } \\
\text { oculocephalic reflexes, } \\
\text { central right facial } \\
\text { paresis, profound } \\
\text { cognitive defects }\end{array}$ & & {$[15]$} \\
\hline 25 & 15 & female & $\begin{array}{l}\text { No cerebral edema in } \\
\text { first } 24 \text { hours on CT. } \\
\text { Multiple small } \\
\text { hematomas, mainly } \\
\text { parieto-occipital, on } \\
\text { day } 12 \text { MRI }\end{array}$ & $\begin{array}{l}\text { First episode diabetes. } \\
\text { Significant } \\
\text { hypotension, } \\
\text { unconscious at } \\
\text { presentation. } \\
\text { Neurologically normal } \\
\text { day } 4 \text {. Bilateral knee } \\
\text { clonus, extensor } \\
\text { plantar response and } \\
\text { peripheral nerve palsies } \\
\text { on day } 7\end{array}$ & & $\begin{array}{l}\text { Decreased platelets } \\
(85,000), \text { normal } \\
\text { coagulation profile }\end{array}$ & {$[23]$} \\
\hline 26 & 11 & female & $\begin{array}{l}\text { Normal MRI. On } \\
\text { autopsy: pin-point } \\
\text { hemorrhages with } \\
\text { ring-and-ball } \\
\text { morphology in } \\
\text { hemispheric white } \\
\text { matter, throughout } \\
\text { brainstem and spinal } \\
\text { cord }\end{array}$ & $\begin{array}{l}\text { First presentation } \\
\text { diabetes, Hypotension, } \\
\text { rapid deterioration in } \\
\text { LOC }\end{array}$ & $\begin{array}{l}\text { Death from renal } \\
\text { complications }\end{array}$ & $\begin{array}{l}\text { Normal } \\
\text { coagulation studies }\end{array}$ & {$[24]$} \\
\hline 27 & 14 & female & $\begin{array}{l}\text { Petechial } \\
\text { hemorrhages in B/L } \\
\text { subcortical white } \\
\text { matter U fibers, genu } \\
\text { of corpus callosum, } \\
\text { posterior limb of } \\
\text { internal capsule, } \\
\text { frontal lobe on MRI }\end{array}$ & $\begin{array}{l}\text { First presentation } \\
\text { diabetes, significant } \\
\text { hypotension. } \\
\text { Unresponsive and } \\
\text { dyspneic }\end{array}$ & $\begin{array}{l}\text { Short term memory } \\
\text { loss, moderate } \\
\text { cognitive deficits }\end{array}$ & $\begin{array}{l}\text { Normal } \\
\text { coagulation studies }\end{array}$ & {$[24]$} \\
\hline 28 & 5 & female & $\begin{array}{l}\text { Hemorrhagic infarct } \\
\text { left thalamus }\end{array}$ & $\begin{array}{l}\text { First presentation } \\
\text { diabetes. Right central } \\
\text { facial palsy, right } \\
\text { hemiplegia, right } \\
\text { babinski sign on day } 7 \\
\text { of treatment }\end{array}$ & $\begin{array}{l}\text { Mild learning } \\
\text { difficulties }\end{array}$ & $\begin{array}{l}\text { normal bleeding } \\
\text { studies, normal } \\
\text { protein } \mathrm{C} \text { and } \mathrm{S} \text { at } \\
\text { time of } \\
\text { hemorrhage }\end{array}$ & {$[25]$} \\
\hline
\end{tabular}

LOC: level of consciousness; B/L: bilateral; tPA: tissue plasminogen activator. 
TABLE 2: Coagulation abnormalities noted in cases of pediatric DKA with stroke and in studies of DKA-associated coagulopathy in adults and children. Pretreatment abnormalities include those noted prior to resolution of biochemical abnormalities. Posttreatment abnormalities include those noted after resolution of biochemical abnormalities.

\begin{tabular}{|c|c|c|c|c|c|}
\hline Factor & Parameter & $\begin{array}{c}\text { Pre-treatment } \\
\text { abnormality }\end{array}$ & $\begin{array}{c}\text { Post-treatment } \\
\text { abnormality }\end{array}$ & $\begin{array}{l}\text { Pediatric versus } \\
\text { adult study }\end{array}$ & Relevant references \\
\hline \multirow{2}{*}{ Platelet } & count & $\sqrt{ }$ & $\sqrt{ }$ & pediatric & {$[5,14,20,23,33]$} \\
\hline & $\begin{array}{l}\text { aggregation/ } \\
\text { activity }\end{array}$ & $\sqrt{ }$ & $\sqrt{ }$ & pediatric, adult & {$[5,35,39]$} \\
\hline Thromboxane $\mathrm{B}_{2}$ & production & & $\sqrt{ }$ & pediatric & {$[14]$} \\
\hline Prothrombin time & & & $\sqrt{ }$ & pediatric & {$[33]$} \\
\hline $\begin{array}{l}\text { Partial } \\
\text { thromboplastin } \\
\text { time } \\
\end{array}$ & levels & & $\sqrt{ }$ & pediatric & {$[33]$} \\
\hline Tissue Factor & levels & $\sqrt{ }$ & & adult & {$[40]$} \\
\hline \multirow{2}{*}{ vWF } & antigen level & $\sqrt{ }$ & $\sqrt{ }$ & pediatric & {$[14,41]$} \\
\hline & activity & $\sqrt{ }$ & & pediatric & {$[41]$} \\
\hline $\begin{array}{l}\text { Factor VIII-vWF } \\
\text { complex }\end{array}$ & levels & & $\sqrt{ }$ & pediatric & {$[14]$} \\
\hline Factor V & levels & & $\sqrt{ }$ & pediatric & {$[5]$} \\
\hline Factor VII & levels & & $\sqrt{ }$ & pediatric & {$[5]$} \\
\hline Factor VIII & levels & & $\sqrt{ }$ & pediatric & {$[5]$} \\
\hline Homocysteine & levels & $\sqrt{ }$ & & pediatric & {$[41]$} \\
\hline Folate & levels & $\sqrt{ }$ & & pediatric & {$[41]$} \\
\hline $\begin{array}{l}\text { Prothrombin } \\
\text { fragment } 1+2\end{array}$ & levels & $\sqrt{ }$ & $\sqrt{ }$ & adult & {$[35]$} \\
\hline $\begin{array}{l}\text { Thrombin- } \\
\text { antithrombin III } \\
\text { complex }\end{array}$ & levels & $\sqrt{ }$ & $\sqrt{ }$ & adult & {$[35]$} \\
\hline Antithrombin III & levels and activity & $\sqrt{ }$ & $\sqrt{ }$ & pediatric, adult & {$[5,20,33,35]$} \\
\hline \multirow{2}{*}{ Protein C } & activity & $\sqrt{ }$ & & pediatric & {$[41]$} \\
\hline & antigen level & $\sqrt{ }$ & $\sqrt{ }$ & pediatric & {$[14,18,41]$} \\
\hline Protein S & Free protein level & $\sqrt{ }$ & & pediatric & {$[42]$} \\
\hline Thrombomodulin & levels & $\sqrt{ }$ & $\sqrt{ }$ & adult & {$[35]$} \\
\hline \multirow{2}{*}{ tPA } & activity & $\sqrt{ }$ & & adult & {$[35,40]$} \\
\hline & antigen level & $\sqrt{ }$ & $\sqrt{ }$ & adult & {$[35,40]$} \\
\hline \multirow{2}{*}{ PAI-1 } & activity & $\sqrt{ }$ & & adult & {$[35,40]$} \\
\hline & antigen level & $\sqrt{ }$ & $\sqrt{ }$ & adult & {$[35,40]$} \\
\hline
\end{tabular}

vWF: von Willebrand Factor; tPA: tissue plasminogen activator; PAI-1: plasminogen activator inhibitor-1.

demonstrated, 1.4-times lower tPA secretion, 2.87-times higher plasminogen activator inhibitor (PAI) activity, and 1.93-times higher PAI antigen level compared to baseline. Combined, these findings suggest that impaired fibrinolytic activity is an early event in DKA. Furthermore, tissue factor (TF), the primary initiator of coagulation, was significantly increased as soon as the insulin infusion was halted. No other coagulation factor demonstrated altered serum levels, and it is not yet clear that TF promotes procoagulant changes in diabetic patients.

An examination of 34 adult patients with uncomplicated DKA demonstrated evidence of endothelial injury, platelet activation, relative hypofibrinolysis, and activation of the coagulation system, even in the absence of clinical signs of thrombosis [35]. Not surprisingly, multivariate analysis indicated that many of the endothelial, clinical, and hemostatic factors were interrelated. Unlike the trial of insulin infusion cessation [40], Ileri and colleagues found that fibrinolytic activity (tPA and plasmin- $\alpha_{2}$-antiplasmin complex levels) was increased both before and during DKA treatment. However, the upregulation was not to a degree expected for the increase in coagulation activity (thrombin-antithrombin III complex and prothrombin fragment $1+2$ levels) at DKA presentation. Not all alterations in the coagulation system are procoagulant. Antithrombin III activity is generally increased in T1DM. In contrast, at DKA presentation, levels are slightly lower than baseline but are still higher than in a control population [35]. 
2.3. Platelet Numbers and Function. Increased platelet activity has been inconsistently demonstrated in children with stroke [5], though no systematic studies of platelet activity and aggregation have been performed in children during DKA. Although platelet counts are generally normal in T1DM, platelet function is enhanced both chronically [38] and during an episode of DKA. Adult volunteers experiencing acute hyperglycemia following an oral glucose challenge demonstrate an acute increase in platelet aggregability [39]. It has been postulated that increased platelet activity may be related to decreased nitric oxide availability reported during episodes of DKA $[52,53]$. Ileri and colleagues found that platelet activation coexisted with DKA and was completely normalized after recovery [35].

2.4. Blood Volume, Flow, and Vascular Reactivity. It has been demonstrated in other conditions that dehydration alone does not account for hypercoagulability [54]. Therefore, although DKA may result in significant fluid losses, other factors such as coagulation system abnormalities, hyperglycemia, acidemia-induced red blood cell rigidity (increased blood viscosity) $[55,56]$, and vasoconstriction induced by hyperglycemia may all have an additive role. The vascular response in hyperglycemia has generally been considered vasoconstrictive, and there is some evidence that this may be related to decreased availability of nitric oxide [53]. However, vascular endothelial growth factor expression is increased by circulating ketones ( $\beta$-hydroxybutyrate), leading to activity of nitric oxide-guanylate cyclase pathway, and therefore vasodilation, in mouse models [57]. Clinically, a transcranial Doppler ultrasound study of 5 children with DKA demonstrated significant vascular dysregulation with vasodilation, decreased cerebral blood flow velocity, and loss of normal cerebral blood flow regulation that only normalized after treatment [58]. Another group found normal to increased cerebral blood flow with impaired cerebral autoregulation during episodes of DKA not associated with overt CE in 6 children [59]. Importantly, none of these studies were able to define the effects that DKA has on local microvascular tone and regulation, and therefore on cerebral oxygen delivery.

\section{Pathogenesis of Hemorrhagic Stroke}

While much of the above discussion has focused on the pathogenesis of thrombosis, it is reasonable to suppose that the pathophysiology of hemorrhagic stroke may involve similar principles to that of hypoperfusion or thrombotic stroke. During an episode of DKA, hemorrhage risk is increased by endovascular perturbation secondary to the proinflammatory state, and to hyperglycemia and acidosis causing oxidative injury $[24,30,31]$, as well as ischemic injury to the vessels from cerebrovascular dysregulation [58, $59]$ or presentation in a shock state $[24,25]$. In a case-control study of 41 adult patients with hemorrhagic stroke, $31 \%$ of diabetics had hemorrhagic conversion of infarcts compared to $18 \%$ of nondiabetic stroke patients [60]. While these were not in the setting of DKA, it does raise the possibility that the aforementioned chronic inflammatory state that exists in diabetes mellitus $[27,36]$ places these patients at higher risk of hemorrhagic conversion of ischemic brain infarction.

DIC has been reported in children with DKA [33]. The consumption of coagulation factors in DIC may predispose to hemorrhage. Four cases of isolated intracerebral DIC, including one case of a 3-month old (Table 1, patient $1)$, have been reported $[13,34]$. DIC was identified on postmortem examination as wide-spread occlusion of small vessels by thrombus, many of which were surrounded by petechial hemorrhage. Furthermore, vascular malformations such as arteriovenous malformations (AVMs), aneurisms, and cavernous malformations that predispose to stroke may be relevant in this patient group. In children who experience hemorrhagic stroke in the absence of diabetes mellitus, vascular malformations are the most commonly experienced risk factor, occurring in $20-85 \%$ in case series [61].

DKA is a proinflammatory condition with vascular endothelial perturbation, and dysregulation of the coagulation system features associated with abnormal levels and activities of several coagulation factors, including platelets, which result in a procoagulant state. Additional factors contributing to the procoagulant state are abnormalities in blood volume, blood viscosity, cerebral autoregulation and blood flow, while vascular injury may be a result of oxidative injury and ischemia related to systemic hypoperfusion, vascular dysregulation, or cerebral edema. Hemorrhagic stroke likely arises secondary to hypoxia and the vascular injury encountered in the oxidative, proinflammatory state of DKA.

\section{Pathology: Ischemic versus Hemorrhagic Stroke}

We identified cases of stroke associated with DKA in children and youth through an exhaustive search of the literature. We searched PubMed for the following terms: ["stroke" or "brain infarction" or "thrombosis"] and ["DKA" or "diabetic ketoacidosis"]. Titles were hand searched for relevance, and where significance was unclear the abstract was read. All appropriate articles were obtained and their reference lists were scanned for other articles of relevance. This iterative process was continued until no further new reports became apparent. We have presented (Table 1) the reported cases of DKA-associated stroke in the following categories: arterial ischemic stroke [4-6, 14-16], cerebral venous thrombosis [16-21], and hemorrhagic stroke [4, 15, 22-25]. Based on the limited investigative modalities available to those compiling the reports, it is likely that our ability to differentiate between stroke etiologies (e.g., arterial ischemia due to hypoperfusion or thrombus, or hemorrhagic stroke with bleeding that arose de novo versus secondary to bleeding within an ischemic injury) is limited. This was clearly illustrated in the case control series described by Muir and colleagues, in which 4 of 23 children with DKA who had CT scanning after developing clinical signs of cerebral edema demonstrated subarachnoid or intraventricular hemorrhage without radiologic edema [62]. Thus we determined that, where hemorrhage was the only radiologic finding, the patient was included in the "hemorrhagic stroke" group. The 4 patients from the latter study were not included in Table 1, as there was 


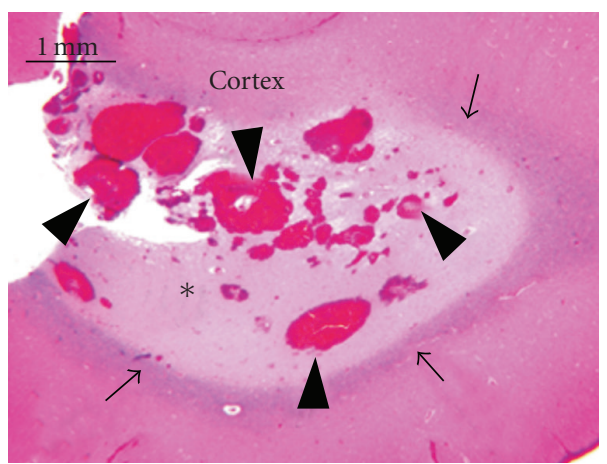

(a)

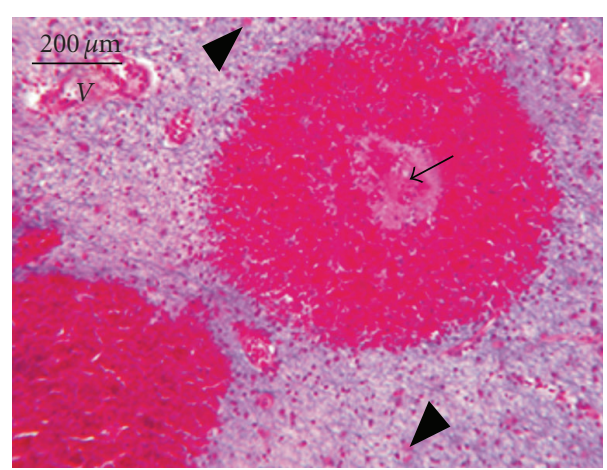

(b)

FIGURE 1: White matter hemorrhages associated with DKA. (a) This low-power view of a gyrus (stained with hematoxylin, eosin, and Luxol fast blue) illustrates multiple small and microscopic hemorrhages (arrowheads) associated with "confluent" pallor (asterisks) of the myelin, a thin layer of preserved subcortical myelin (arrows), and normal cortex. (b) A vessel, labeled $V$, is identified for reference. Stained with Luxol fast blue, hematoxylin, and eosin (which stains myelin blue). Central hemorrhage was formed by perivascular necrosis (arrow), a concentric ring of red blood cells, and diffusely rarefied white matter that is speckled with eosinophilic astrocytes (arrowheads). Figure 1 reproduced with permission from Pediatrics, volume 126, page 1543, copyright 2007 by the AAP.

no specific individual information given. Where multiple small hemorrhages occurred in the setting of clear thrombus or emboli [13], the patient was included in the "arterial ischemic stroke" group. Cases of venous sinus thrombosis with relevant clinical findings in the acute phase, but with no long-term clinical or radiologic sequelae [16], were included, as they represent acute intracerebral events associated with DKA.

The pathologic tissue findings of a patient having experienced acute cerebral infarction related to an episode of DKA are not expected to be different from those of a nondiabetic child who has had a stroke. Two patients (patients 26 and 27) had hemorrhagic stroke that was characterized histologically by a ring-and-ball morphology (Figure 1) [24]. Widespread small vessel occlusion, diagnosed on autopsy, has also been described [13]. As stroke itself may cause cerebral edema, it becomes difficult to ascertain whether cerebral edema in DKA is the cause or an effect of acute cerebral infarction. Table 1 details the course of four patients $(2,3,9,24)$ who had signs of raised intracranial pressure and may have suffered cerebral hypoperfusion and infarction as a complication of DKA-CE. Patients 2, 3, 6, 8, and 28 had infarction limited to areas supplied by the posterior cerebral artery and anterior choroidal arteries (the areas most susceptible to damage following transtentorial herniation), presumably secondary to raised intracranial pressure. However, some of this group lacked clinical signs or radiological evidence (patients $6,8,28$ ) of $\mathrm{CE}$, raising the possibility of a primary thrombotic or hemorrhagic event. Patient 1 had signs of DKA-CE but also clear thrombus formation on autopsy [13].

Two patients were found to have thrombophilic conditions. Patient 14 developed a DVT prior to presentation with central nervous system complications of DKA. MRI demonstrated acute infarctions in multiple areas of the brain, and examination identified heterozygosity for Factor V Leiden [18]. Patient 18, who developed thrombosis of multiple cerebral venous sinuses, was found to have heterozygous mutation of the prothrombin gene (G20210A) [21].

\section{Evaluation}

The clinical presentation of stroke as a focal neurologic deficit should pose little diagnostic problem. Of greater difficulty is differentiating global neurological impairment in DKA patients from severe acidosis, DKA-CE, or primary stroke. Among the patients presented in Table 1, only 8 (patients 10,11, 12, 15, 17, 23, 25, 28) had focal neurologic signs. The remainder presented with nonspecific signs consistent with the global dysfunction seen in DKA-CE. As the presentation of CE and primary stroke in DKA can be so similar, it is imperative that the clinician have a high index of suspicion for stroke. Early imaging is warranted once the patient is stabilized in order to optimize management.

The best modality for identification of the ischemia associated with stroke is magnetic resonance imaging (MRI) with perfusion- and/or diffusion-weighted imaging, which have sensitivity nearing 100\% [63]. Computed tomography (CT) may be used to rule out CE, hemorrhage or abscess, though the sensitivity for identification of ischemic infarction in the acute phase is only 50\% [63]. Additionally, it is agreed that CT may miss cases of cerebral edema, though its sensitivity for identifying elevated intracranial pressure has been reported at $99.1 \%$ [64]. Cerebral angiography is the gold standard for assessment of the cerebral vasculature, although MR angiography (MRA) is able to detect large vascular lesions effectively [65], with the benefit of being noninvasive. CT angiography, though it requires injection of contrast, may be used to evaluate the cerebral circulation early in stroke evolution [66]. In cases where neither MRA nor MRI defines suspected pathology in a distal artery, cerebral angiography may be considered.

All patients with suspected intracranial pathology should have a coagulation screen performed. In cases of clear 
thrombosis or hemorrhage, more detailed analysis of the hemostatic system is warranted. Identification of the exact histopathology may be done with tissue biopsy [24], though this carries many risks and is not recommended as a matter of course.

\section{Management}

Fully evidence-based management guidelines for children experiencing acute ischemic or hemorrhagic infarction do not exist and have been extrapolated from adult data. Admission to the critical care unit and close monitoring is appropriate for any patient with suspected or proven central nervous system (CNS) complication of acute DKA. Unless diffuse CE can be absolutely excluded, or another clear cause is present, emergency management for CNS complications of DKA should prioritize the treatment for cerebral edema. Although early reactive treatment for CE appears beneficial $[4,67]$, it was stated in 1990 that intracerebral complications do not often come with warning signs, and early intervention measures are frequently unsuccessful in preventing complications; so prevention of DKA is the most effective method of preventing complications [4]. In the intervening 20 years, this generally remains true, although there may be some alternative management options for thrombus causing stroke.

Children with stroke should receive aggressive treatment for fever, infection, and seizures [68]. For all forms of stroke, recommendations are for early mobilization and rehabilitation. Current guidelines do not support the use of thrombolytics in pediatric arterial ischemic stroke [69], although thrombotic stroke associated with an episode of DKA is not addressed specifically. Furthermore, multiple case reports document use of thrombolytics treatment associated with good outcome in children with acute ischemic cerebral infarction [70-72], including thrombolysis used successfully up to 36 hours after onset of symptoms [73, 74]. Safe, acute thrombolysis with recombinant tPA was reported for a 10 year-old child with cerebral venous sinus thrombosis that occurred 3 days after onset of DKA, who had complete recovery [21]. However, caution is warranted as the risks of thrombolysis and the optimal tPA doses in children have not been quantified. In light of the lack of evidence or strength of recommendations, it seems prudent that management of arterial ischemic stroke in association with DKA be considered on a case-by-case basis and in consultation with stroke experts.

Beyond the acute phase of ischemic or thrombotic stroke it has generally been agreed that anticoagulation with heparin [75, 76] may be appropriate for pediatric patients who have already experienced arterial ischemic stroke or cerebral venous thrombosis, independent of T1DM and DKA. International guidelines recommend the use of low molecular weight or unfractionated heparin initially, followed by warfarin therapy for 3-6 months [75-77]. There is no clear guidance on the management of children who have experienced hemorrhagic stroke. Adult guidelines suggest that extremely high blood pressures be reduced cautiously and recombinant factor VIIa has shown some promise in decreasing recurrence but is only recommended within clinical trials. However, these guidelines refer to different etiologies for intracranial hemorrhage than are seen in children or in the DKA population. Large hemorrhages compromising neurovascular structures may warrant surgical decompression.

Prophylactic systemic anticoagulation has been suggested for patients in DKA $[13,35,78]$. However, anticoagulation is not addressed in international consensus statements on the management of DKA $[1,79,80]$. In light of the risk of hemorrhagic stroke and the unknown incidence of stroke in pediatric DKA, a broad recommendation for prophylactic anticoagulation cannot be supported at this time.

\section{Outcome}

Stroke outcome depends on the cerebral regions affect and the extent of the injury. As demonstrated from the case series (Table 1), the majority of children with DKA-associated stroke reported have some form of residual neurologic deficit with death or persistent vegetative state as the outcome in 8 of 28 patients $(29 \%)$ and full recovery seen in only 4 of 28 cases $(14 \%)$.

\section{Conclusions}

Stroke in DKA is uncommon but life-threatening. DKA may be considered an inflammatory condition with vascular endothelial perturbation and dysfunction of the coagulation system. Multiple causes of thrombus have been postulated and studies show several contributing mechanisms. Hemorrhagic infarctions are rare and may be multifactorial but must be considered a risk. Management for CNS complications of DKA should prioritize the treatment for cerebral edema. As the initial presentation of pediatric stroke can be subtle and may be confused with DKA-CE, early imaging for any young person with neurologic deterioration in association with an episode of DKA is imperative following emergency treatment for $\mathrm{CE}$ and stabilization.

\section{References}

[1] J. Wolfsdorf, N. Glaser, and M. A. Sperling, "Diabetic ketoacidosis in infants, children, and adolescents: a consensus statement from the American Diabetes Association," Diabetes Care, vol. 29, no. 5, pp. 1150-1159, 2006.

[2] J. Scibilia, D. Finegold, J. Dorman, D. Becker, and A. Drash, "Why do children with diabetes die?" Acta Endocrinologica. Supplementum, vol. 279, pp. 326-333, 1986.

[3] J. A. Edge, M. E. Ford-Adams, and D. B. Dunger, "Causes of death in children with insulin dependent diabetes 1990-96," Archives of Disease in Childhood, vol. 81, no. 4, pp. 318-323, 1999. 
[4] A. L. Rosenbloom, "Intracerebral crises during treatment of diabetic ketoacidosis," Diabetes Care, vol. 13, no. 1, pp. 22-33, 1990.

[5] T. F. Roe, T. O. Crawford, K. R. Huff, G. Costin, F. R. Kaufman, and M. D. Nelson, "Brain infarction in children with diabetic ketoacidosis," Journal of Diabetes and its Complications, vol. 10, no. 2, pp. 100-108, 1996.

[6] J. Ho, D. Pacaud, M. D. Hill, C. Ross, L. Hamiwka, and J. K. Mah, "Diabetic ketoacidosis and pediatric stroke," Canadian Medical Association Journal, vol. 172, no. 3, pp. 327-328, 2005.

[7] J. A. Edge, M. M. Hawkins, D. L. Winter, and D. B. Dunger, "The risk and outcome of cerebral oedema developing during diabetic ketoacidosis," Archives of Disease in Childhood, vol. 85, no. 1, pp. 16-22, 2001.

[8] S. E. Lawrence, E. A. Cummings, I. Gaboury, and D. Daneman, "Population-based study of incidence and risk factors for cerebral edema in pediatric diabetic ketoacidosis," Journal of Pediatrics, vol. 146, no. 5, pp. 688-692, 2005.

[9] A. L. Rosenbloom, "Hyperglycemic crises and their complications in children," Journal of Pediatric Endocrinology and Metabolism, vol. 20, no. 1, pp. 5-18, 2007.

[10] S. Likitmaskul, J. Santiprabhob, P. Sawathiparnich, N. Numbenjapon, and K. Chaichanwatanakul, "Clinical pictures of type 2 diabetes in Thai children and adolescents is highly related to features of metabolic syndrome," Journal of the Medical Association of Thailand, vol. 88, supplement 8, pp. S169-S175, 2005.

[11] R. L. Sacco, "Risk factors and outcomes for ischemic stroke," Neurology, vol. 45, no. 2, pp. S10-14, 1995.

[12] . The Emerging Risk Factors Collaboration, "Diabetes mellitus, fasting blood glucose concentration, and risk of vascular disease: a collaborative meta-analysis of 102 prospective studies," The Lancet, vol. 375, no. 9733, pp. 2215-2222, 2010.

[13] W. R. Timperley, F. E. Preston, and J. D. Ward, "Cerebral intravascular coagulation in diabetic ketoacidosis," The Lancet, vol. 1, no. 7864, pp. 952-956, 1974.

[14] R. K. Kanter, M. Oliphant, J. J. Zimmerman, and M. J. Stuart, "Arterial thrombosis causing cerebral edema in association with diabetic ketoacidosis," Critical Care Medicine, vol. 15, no. 2, pp. 175-176, 1987.

[15] B. Rogers, I. Sills, M. Cohen, and F. G. Seidel, "Diabetic Ketoacidosis: neurologic collapse during treatment followed by severe developmental morbidity," Clinical Pediatrics, vol. 29, no. 8, pp. 451-456, 1990.

[16] J. Ho, J. K. Mah, M. D. Hill, and D. Pacaud, "Pediatric stroke associated with new onset type 1 diabetes mellitus: case reports and review of the literature," Pediatric Diabetes, vol. 7, no. 2, pp. 116-121, 2006.

[17] S. Keane, A. Gallagher, S. Ackroyd, M. A. McShane, and J. A. Edge, "Cerebral venous thrombosis during diabetic ketoacidosis," Archives of Disease in Childhood, vol. 86, no. 3, pp. 204-206, 2002.

[18] A. L. Rosenbloom, "Fatal cerebral infarctions in diabetic ketoacidosis in a child with previously unknown heterozygosity for factor V Leiden deficiency," Journal of Pediatrics, vol. 145, no. 4, pp. 561-562, 2004.

[19] K. De Keyzer, K. Paemeleire, M. De Clerck, D. Peeters, and J. L. De Reuck, "Diabetic ketoacidosis presenting as a cerebral venous sinus thrombosis," Acta Neurologica Belgica, vol. 104, no. 3, pp. 117-120, 2004.

[20] M. J. Sasiadek, D. Sosnowska-Pacuszko, M. Zielinska, and T. Turek, "Cerebral venous thrombosis as a first presentation of diabetes," Pediatric Neurology, vol. 35, no. 2, pp. 135-138, 2006.
[21] M. Zerah, R. Patterson, I. Hansen, M. Briones, J. Dion, and B. Renfroe, "Resolution of severe sinus vein thrombosis with super selective thrombolysis in a pre-adolescent with diabetic ketoacidosis and a prothrombin gene mutation," Journal of Pediatric Endocrinology and Metabolism, vol. 20, no. 6, pp. 725-731, 2007.

[22] V. L. Atluru, "Spontaneous intracerebral hematomas in juvenile diabetic ketoacidosis," Pediatric Neurology, vol. 2, no. 3, pp. 167-169, 1986.

[23] S. L. Atkin, A. M. Coady, D. Horton, N. Sutaria, L. Sellars, and C. Walton, "Multiple cerebral haematomata and peripheral nerve palsies associated with a case of juvenile diabetic ketoacidosis," Diabetic Medicine, vol. 12, no. 3, pp. 267-270, 1995.

[24] F. H. Mahmud, D. A. Ramsay, S. D. Levin, R. N. Singh, T. Kotylak, and D. D. Fraser, "Coma with diffuse white matter hemorrhages in juvenile diabetic ketoacidosis," Pediatrics, vol. 120, no. 6, pp. e1540-e1546, 2007.

[25] J. J. Lin, K. L. Lin, H. S. Wang, A. M. C. Wong, and S. H. Hsia, "Occult infarct with acute hemorrhagic stroke in juvenile diabetic ketoacidosis," Brain and Development, vol. 30, no. 1, pp. 91-93, 2008.

[26] W. H. Hoffman, C. L. Burek, J. L. Waller, L. E. Fisher, M. Khichi, and L. B. Mellick, "Cytokine response to diabetic ketoacidosis and its treatment," Clinical Immunology, vol. 108, no. 3, pp. 175-181, 2003.

[27] C. G. Schalkwijk, D. C. W. Poland, W. Van Dijk et al., "Plasma concentration of C-reactive protein is increased in Type I diabetic patients without clinical macroangiopathy and correlates with markers of endothelial dysfunction: evidence for chronic inflammation," Diabetologia, vol. 42, no. 3, pp. 351-357, 1999.

[28] R. R. Dalton, W. H. Hoffman, G. G. Passmore, and S. L. A. Martin, "Plasma C-reactive protein levels in severe diabetic ketoacidosis," Annals of Clinical and Laboratory Science, vol. 33, no. 4, pp. 435-442, 2003.

[29] W. H. Hoffman, C. D. Cudrici, E. Zafranskaia, and H. Rus, "Complement activation in diabetic ketoacidosis brains," Experimental and Molecular Pathology, vol. 80, no. 3, pp. 283288, 2006.

[30] A. Ceriello, D. Giugliano, A. Quatraro, E. Marchi, M. Barbanti, and P. Lefebvre, "Possible role for increased C4b-bindingprotein level in acquired protein $\mathrm{S}$ deficiency in type I diabetes," Diabetes, vol. 39, no. 4, pp. 447-449, 1990.

[31] S. K. Jain, R. McVie, and J. A. Bocchini Jr., "Hyperketonemia (ketosis), oxidative stress and type 1 diabetes," Pathophysiology, vol. 13, no. 3, pp. 163-170, 2006.

[32] W. H. Hoffman, J. P. Locksmith, E. M. Burton et al., "Interstitial pulmonary edema in children and adolescents with diabetic ketoacidosis," Journal of Diabetes and its Complications, vol. 12, no. 6, pp. 314-320, 1998.

[33] R. Bonfanti, E. Bognetti, F. Meschi, S. Medaglini, A. D’Angelo, and G. Chiumello, "Disseminated intravascular coagulation and severe peripheral neuropathy complicating ketoacidosis in a newly diagnosed diabetic child," Acta Diabetologica, vol. 31, no. 3, pp. 173-174, 1994.

[34] F. Shanahan, G. Mortimer, and M. I. Drury, "Cerebral intravascular coagulation complicating diabetic ketoacidosis," Irish Journal of Medical Science, vol. 150, no. 5, pp. 156-157, 1981.

[35] N. Ş. Ileri, Y. Büyükaşik, S. Karaahmetoğlu et al., "Evaluation of the haemostatic system during ketoacidotic deterioration of diabetes mellitus," Haemostasis, vol. 29, no. 6, pp. 318-325, 2000. 
[36] M. Romano, M. Pomilio, S. Vigneri et al., "Endothelial perturbation in children and adolescents with type 1 diabetes: association with markers of the inflammatory reaction," Diabetes Care, vol. 24, no. 9, pp. 1674-1678, 2001.

[37] N. Doğruel, B. Kirel, Y. Akgün, and T. Us, "Serum soluble endothelial-cell specific adhesion molecules in children with insulin-dependent diabetes mellitus," Journal of Pediatric Endocrinology and Metabolism, vol. 14, no. 3, pp. 287-293, 2001.

[38] M. E. Carr, "Diabetes mellitus: a hypercoagulable state," Journal of Diabetes and its Complications, vol. 15, no. 1, pp. 44-54, 2001.

[39] T. Sakamoto, H. Ogawa, H. Kawano et al., "Rapid change of platelet aggregability in acute hyperglycemia. Detection by a novel laser-light scattering method," Thrombosis and Haemostasis, vol. 83, no. 3, pp. 475-479, 2000.

[40] A. Iorio, M. O. Federici, E. Mourvaki et al., "Impaired endothelial antithrombotic activity following short-term interruption of continous subcutaneous insulin infusion in type I diabetic patients," Thrombosis and Haemostasis, vol. 98, no. 3, pp. 635-641, 2007.

[41] G. F. Carl, W. H. Hoffman, G. G. Passmore et al., "Diabetic ketoacidosis promotes a prothrombotic state," Endocrine Research, vol. 29, no. 1, pp. 73-82, 2003.

[42] J. M. Worly, J. D. Fortenberry, I. Hansen, C. R. Chambliss, and J. Stockwell, "Deep venous thrombosis in children with diabetic ketoacidosis and femoral central venous catheters," Pediatrics, vol. 113, no. 1, pp. e57-e60, 2004.

[43] J. A. Gutierrez, R. Bagatell, M. P. Samson, A. A. Theodorou, and R. A. Berg, "Femoral central venous catheter-associated deep venous thrombosis in children with diabetic ketoacidosis," Critical Care Medicine, vol. 31, no. 1, pp. 80-83, 2003.

[44] C. Beck, J. Dubois, A. Grignon, J. Lacroix, and M. David, "Incidence and risk factors of catheter-related deep vein thrombosis in a pediatric intensive care unit: a prospective study," Journal of Pediatrics, vol. 133, no. 2, pp. 237-241, 1998.

[45] T. C. Vukovich and G. Schernthaner, "Decreased protein $\mathrm{C}$ levels in patients with insulin-dependent type I diabetes mellitus," Diabetes, vol. 35, no. 5, pp. 617-619, 1986.

[46] G. N. Welch and J. Loscalzo, "Homocysteine and atherothrombosis," New England Journal of Medicine, vol. 338, no. 15, pp. 1042-1050, 1998.

[47] G. M. Rodgers and M. T. Conn, "Homocysteine, an atherogenic stimulus, reduces protein $\mathrm{C}$ activation by arterial and venous endothelial cells," Blood, vol. 75, no. 4, pp. 895-901, 1990.

[48] M. Greaves, C. Pickering, and G. Knight, "Changes in the factor VIII complex in diabetic ketoacidosis: evidence of endothelial cell damage?" Diabetologia, vol. 30, no. 3, pp. 160$165,1987$.

[49] R. C. Paton, "Haemostatic changes in diabetic coma," Diabetologia, vol. 21, no. 3, pp. 172-177, 1981.

[50] M. Mirshahi, J. Soria, C. Soria, O. Bertrand, M. Mirshahi, and A. Basdevant, "Glycosylation of human fibrinogen and fibrin in vitro. Its consequences on the properties of fibrin(ogen)," Thrombosis Research, vol. 48, no. 3, pp. 279-289, 1987.

[51] M. E. Carr and B. M. Alving, "Effect of fibrin structure on plasmin-mediated dissolution of plasma clots," Blood Coagulation and Fibrinolysis, vol. 6, no. 6, pp. 567-573, 1995.

[52] M. R. Adams, C. J. Forsyth, W. Jessup, J. Robinson, and D. S. Celermajer, "Oral L-arginine inhibits platelet aggregation but does not enhance endothelium-dependent dilation in healthy young men," Journal of the American College of Cardiology, vol. 26, no. 4, pp. 1054-1061, 1995.
[53] D. Giugliano, R. Marfella, L. Coppola et al., "Vascular effects of acute hyperglycemia in humans are reversed by L- arginine: evidence for reduced availability of nitric oxide during hyperglycemia," Circulation, vol. 95, no. 7, pp. 17831790, 1997.

[54] D. L. Sackett, "Possible risk factors in the development of venous thrombosis. Gastrointestinal disease, infection and dehydration," The Milbank Memorial Fund quarterly, vol. 50, supplement 2, pp. 105-122, 1972.

[55] L. A. Evan-Wong, R. J. L. Davidson, and J. M. Stowers, "Alterations in erythrocytes in hyperosmolar diabetic decompensation: a pathophysiological basis for impaired blood flow and for an improved design of fluid therapy," Diabetologia, vol. 28, no. 10, pp. 739-742, 1985.

[56] E. M. Sevick and R. K. Jain, "Effect of red blood cell rigidity on tumor blood flow: increase in viscous resistance during hyperglycemia," Cancer Research, vol. 51, no. 10, pp. 27272730, 1991.

[57] C. M. Isales, L. Min, and W. H. Hoffman, "Acetoacetate and $\beta$ hydroxybutyrate differentially regulate endothelin-1 and vascular endothelial growth factor in mouse brain microvascular endothelial cells," Journal of Diabetes and its Complications, vol. 13, no. 2, pp. 91-97, 1999.

[58] W. H. Hoffman, R. M. Pluta, A. Q. Fisher, M. B. Wagner, and J. A. Yanovski, "Transcranial Doppler ultrasound assessment of intracranial hemodynamics in children with diabetic ketoacidosis," Journal of Clinical Ultrasound, vol. 23, no. 9, pp. 517523, 1995.

[59] J. S. Roberts, M. S. Vavilala, K. A. Schenkman, D. Shaw, L. D. Martin, and A. M. Lam, "Cerebral hyperemia and impaired cerebral autoregulation associated with diabetic ketoacidosis in critically ill children," Critical Care Medicine, vol. 34, no. 8, pp. 2217-2223, 2006.

[60] E. Beghi, G. Bogliun, G. Cavaletti et al., "Hemorrhagic infarction: risk factors, clinical and tomographic features, and outcome. A case-control study," Acta Neurologica Scandinavica, vol. 80, no. 3, pp. 226-231, 1989.

[61] J. K. Lynch, "Cerebrovascular disorders in children," Current Neurology and Neuroscience Reports, vol. 4, no. 2, pp. 129-138, 2004.

[62] A. B. Muir, R. G. Quisling, M. C. K. Yang, and A. L. Rosenbloom, "Cerebral edema in childhood diabetic ketoacidosis: natural history, radiographic findings, and early identification," Diabetes Care, vol. 27, no. 7, pp. 1541-1546, 2004.

[63] N. Hoggard, I. D. Wilkinson, and P. D. Griffiths, "The imaging of ischaemic stroke," Clinical Radiology, vol. 56, no. 3, pp. 171$183,2001$.

[64] W. Hirsch, R. Beck, C. Behrmann, A. Schobess, and R. P. Spielmann, "Reliability of cranial CT versus intracerebral pressure measurement for the evaluation of generalised cerebral oedema in children," Pediatric Radiology, vol. 30, no. 7, pp. 439-443, 2000.

[65] B. Husson, G. Rodesch, P. Lasjaunias, M. Tardieu, and G. Sébire, "Magnetic resonance angiography in childhood arterial brain infarcts: a comparative study with contrast angiography," Stroke, vol. 33, no. 5, pp. 1280-1285, 2002.

[66] D. S. Atkinson, "Computed Tomography of Pediatric Stroke," Seminars in Ultrasound, CT and MRI, vol. 27, no. 3, pp. 207$218,2006$.

[67] B. Franklin, J. Liu, and F. Ginsberg-Fellner, "Cerebral edema and ophthalmoplegia reversed by mannitol in a new case of insulin-dependent diabetes mellitus," Pediatrics, vol. 69, no. 1, pp. 87-90, 1982. 
[68] M. Lopez-Vicente, S. Ortega-Gutierrez, C. Amlie-Lefond, and M. T. Torbey, "Diagnosis and Management of Pediatric Arterial Ischemic Stroke," Journal of Stroke and Cerebrovascular Diseases, vol. 19, no. 3, pp. 175-183, 2010.

[69] D. Eleftheriou and V. Ganesan, "Controversies in childhood arterial ischemic stroke and cerebral venous sinus thrombosis," Expert Review of Cardiovascular Therapy, vol. 7, no. 7, pp. 853-861, 2009.

[70] R. Belvís, "Thrombolysis for acute stroke in pediatrics," Stroke, vol. 38, no. 6, pp. 1722-1723, 2007.

[71] M. I. Shuayto, J. I. Lopez, and F. Greiner, "Administration of intravenous tissue plasminogen activator in a pediatric patient with acute ischemic stroke," Journal of Child Neurology, vol. 21, no. 7, pp. 604-606, 2006.

[72] S. L. Benedict, O. K. Ni, P. Schloesser, K. S. White, and J. F. Bale, "Intra-arterial thrombolysis in a 2-year-old with cardioembolic stroke," Journal of Child Neurology, vol. 22, no. 2, pp. 225-227, 2007.

[73] C. Cognard, A. Weill, S. Lindgren, M. Piotin, L. Castaings, and J. Moret, "Basilar artery occlusion in a child: "Clot angioplasty” followed by thrombolysis," Child's Nervous System, vol. 16, no. 8, pp. 496-500, 2000.

[74] A. Kirton, J. H. Wong, J. Mah et al., "Successful endovascular therapy for acute basilar thrombosis in an adolescent," Pediatrics, vol. 112, no. 3, part 1, pp. e248-e251, 2003.

[75] P. Monagle, E. Chalmers, A. Chan et al., "Antithrombotic therapy in neonates and children: American College of Chest Physicians evidence-based clinical practice guidelines (8th edition)," Chest, vol. 133, no. 6, supplement, pp. 887S-968S, 2008.

[76] E. S. Roach, M. R. Golomb, R. Adams et al., "Management of stroke in infants and children: a scientific statement from a special writing group of the american heart association stroke council and the council on cardiovascular disease in the young," Stroke, vol. 39, no. 9, pp. 2644-2691, 2008.

[77] Paediatric Stroke Working Group, Stroke in Childhood: Clinical Guidelines for Diagnosis, Management and Rehabilitation, Royal College of Physicians, London ,UK, 2004.

[78] J. Burzynski, J. Ho, D. Pacaud, and J. Mah, "DKA and thrombosis," Canadian Medical Association Journal, vol. 173, no. 2, pp. 132-133, 2005.

[79] D. B. Dunger, M. A. Sperling, C. L. Acerini et al., "European Society for Paediatric Endocrinology/Lawson Wilkins Pediatric Endocrine Society consensus statement on diabetic ketoacidosis in children and adolescents," Pediatrics, vol. 113, no. 2, pp. e133-e140, 2004.

[80] M. L. Lawson, D. Pacaud, S. E. Lawrence, D. Daneman, and H. J. Dean, "2003 Canadian clinical practice guidelines for the management of diabetes in children and adolescents," Paediatrics and Child Health, vol. 10, supplement A, pp. 5A16A, 2005. 


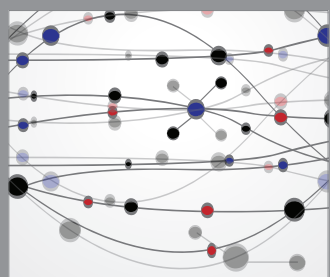

The Scientific World Journal
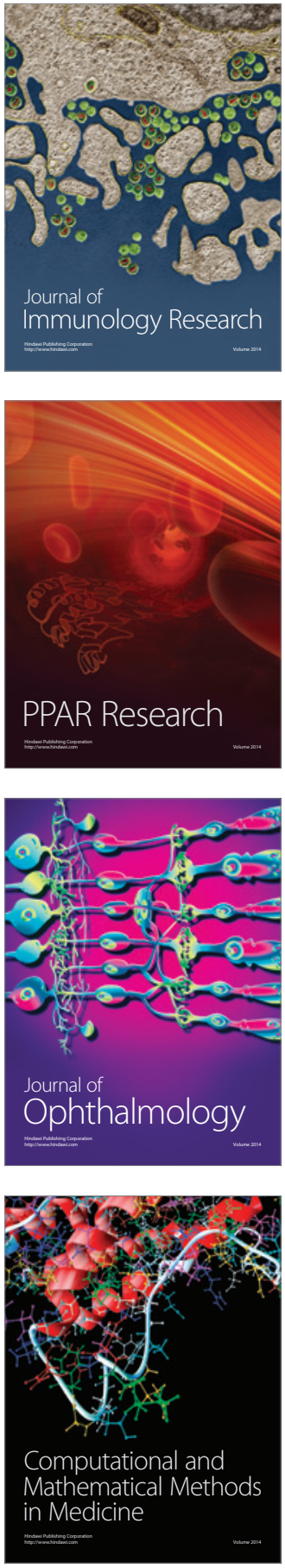

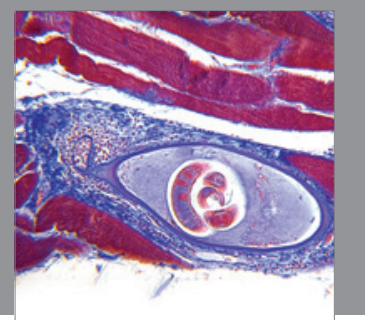

Gastroenterology

Research and Practice
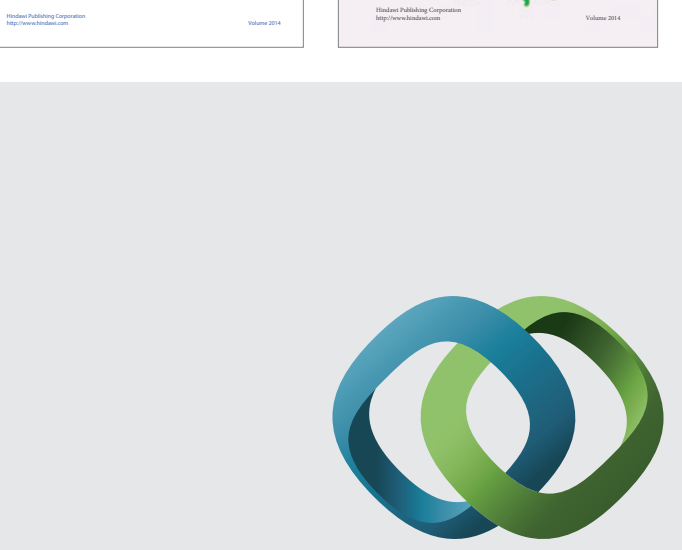

\section{Hindawi}

Submit your manuscripts at

http://www.hindawi.com
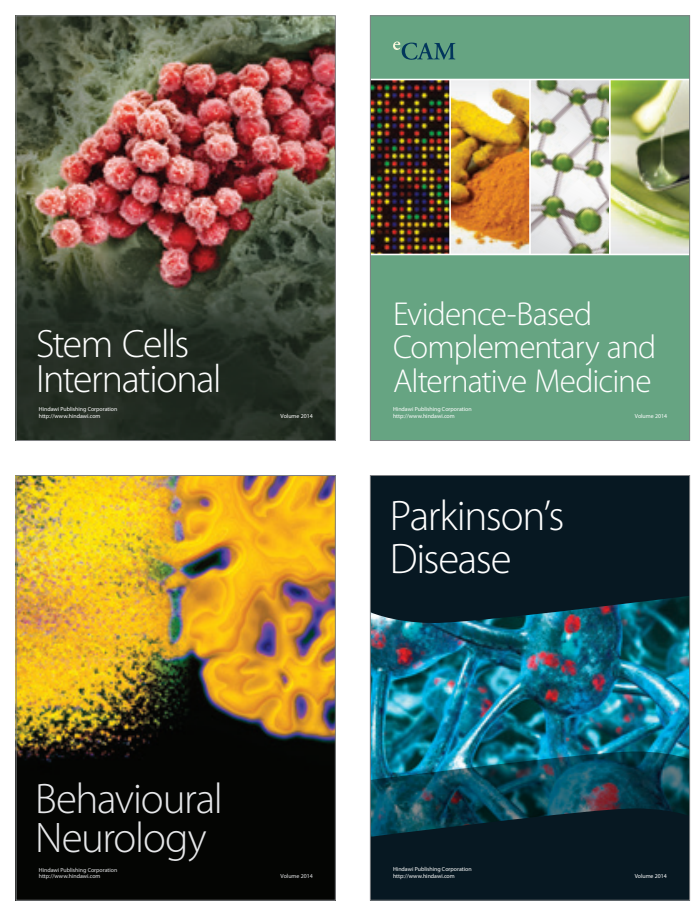

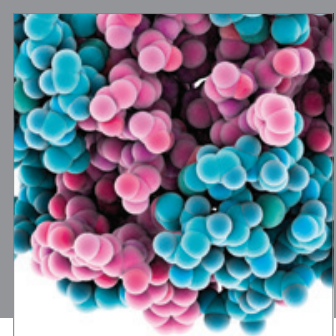

Journal of
Diabetes Research

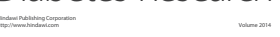

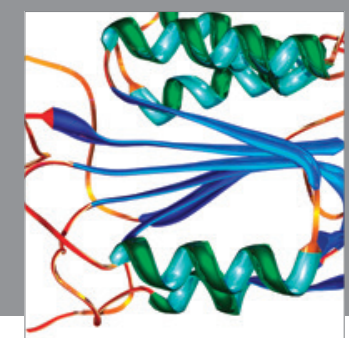

Disease Markers
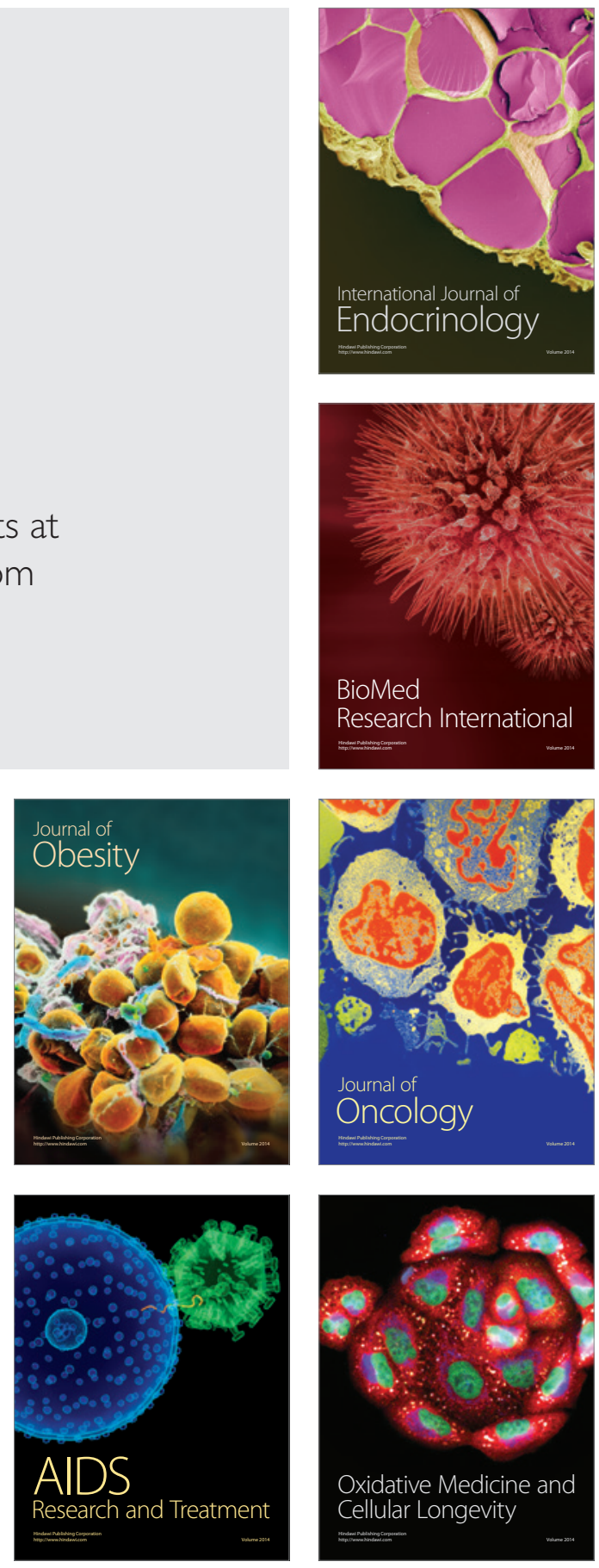